\title{
Digital Darkfield Analysis of Lattice Fringe Images with ImageJ
}

\section{P. Fraundorf}

Center for NanoScience/Physics \& Astronomy, University of Missouri StL, St. Louis MO 63121 USA.

Digital-darkfield analysis involves the spatial mapping of selected frequencies across a digitized image, much as analog (e.g. electron-optical) darkfield-imaging allows one to locate crystals responsible for a given diffraction spot. This analysis involves a type of image decomposition which, like wavelets, is intermediate between direct and reciprocal (spatial-frequency) space [1]. Digital-darkfield analysis is of particular use on images that have well defined spatial-frequencies in them, like lattice-fringe images of atomic-planes encountered edge-on by high-energy electrons in crystalline materials [2-10].

A set of java plugins is posted on the ImageJ wiki at http://imagejdocu.tudor.lu/, along with macros for putting them to use. The java plugins include routines for quantitatively converting complex-number arrays to and from RGB images with pixel-intensity proportional to coefficient log-amplitude and pixel-hue linked to coefficient phase. These plugins may also be used by hue-maximization routines, to be described elsewhere.

Some of the routines allow the user to place a circular aperture in the power spectrum of an image, from which periodicity amplitude and phase-gradient (strain) maps are calculated, labeled, and displayed. A periodicity-amplitude map is shown (top right panel) in Fig. 1, for one of the many periodicities in the power spectrum (top center panel) of an electron phase-contrast image of some $\mathrm{WC}_{1-\mathrm{x}}$ nanocrystals [11] (top left panel). Fig. 2 shows periodicity amplitude banding (left) with twice the frequency of ordinary thickness fringes, as well as the periodicity phase-reversals (right) expected to occur therewith.

Other routines let the user tile frequency space with an array of tiny darkfield (plus one brightfield) images, yielding a tableau containing one direct-space image formed by the spatial-periodicities found in each frequency-space tile. This darkfield-tableau strategy has helped find patterns, like icosahedral twin"bow-ties" and "butterflies" in images of randomly-oriented nanoparticles [12-13].

[1] P. Fraundorf (2004) arXiv:cond-mat/040301 http://arxiv.org/abs/cond-mat/0403017

[2] P. Fraundorf and Lu Fei (2004) Microscopy and MicroAnalysis 10:s2, 300-301.

[3] W. Qin and P. Fraundorf (2005) Microscopy and MicroAnalysis 11:s2, p562-563

[4] P. Fraundorf, Jinfeng Wang, Eric Mandell and Martin Rose (2006) M\&M 12:s2, 1010-1011.

[5] Martin Rose and P. Fraundorf (2006) Microscopy and Microanalysis 12:s2, 1008-1009.

[6] P. Fraundorf, J. Liu and E. Mandell (2007) 13:s2, 992-993.

[7] P. Fraundorf and Somik Mukherjee (2013), Microscopy and MicroAnalysis 19:s2, 1590-1591.

[8] Mukherjee S, Ramalingam B, Griggs L, Hamm S, Baker GA, Fraundorf P, Sengupta S,

Gangopadhyay S. (2012) Nanotechnology 23(48):485405

[9] P. Fraundorf, Wentao Qin, P. Moeck and Eric Mandell (2005) J. Applied Physics 98:114308

[10] P. Wang, A. L. Bleloch, U. Falke and P. J. Goodhew (2006) Ultramicroscopy 106:277-283.

[1 1] Wentao Qin and P. Fraundorf (2003) Ultramicroscopy 94:3-4, 246-262.

[12] P. Fraundorf, Jinfeng Wang, Eric Mandell and Martin Rose (2006) M\&M 12:s2, 1010-1011

[13] P. Fraundorf and Chris Bishop (2013) Microscopy and MicroAnalysis 19:s2, 1804-1805. 


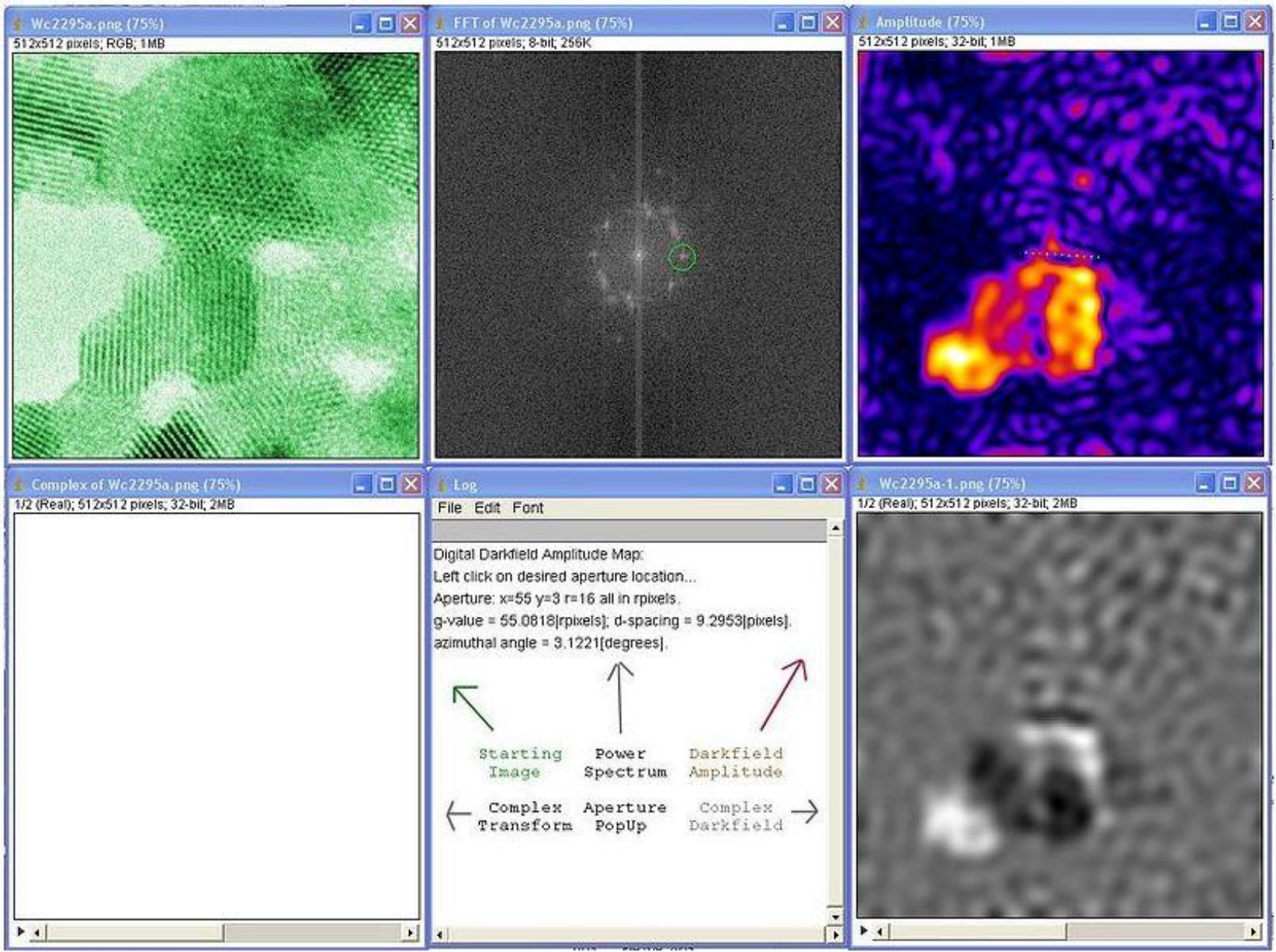

Figure 1. In this example, a crystal with lattice fringes in only one direction is shown (top right) to be responsible for the circled diffraction-spot in the image power-spectrum (top center).

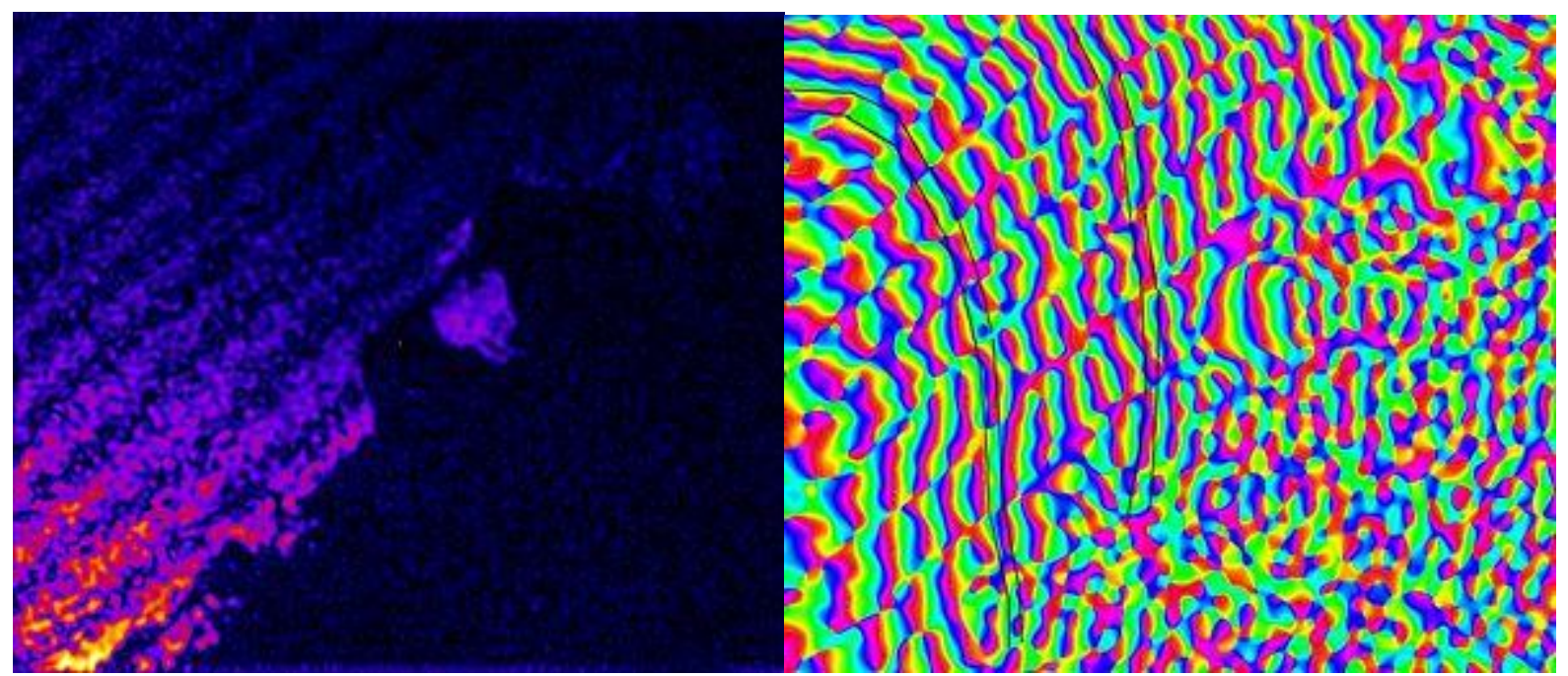

Figure 2: Si-111 darkfield amplitude map of a 4024x3443 pixel lattice image (left), is shown in the darkfield phase image (right) to have phase-inversions expected at thickness-fringe maxima $\&$ minima. 